\title{
Parallel representation of stimulus identity and intensity in a dual pathway model inspired by the olfactory system of the honeybee
}

\author{
Michael Schmuker ${ }^{1,2,3}$, Nobuhiro Yamagata ${ }^{2,4}{ }^{\dagger}$, Martin Paul Nawrot $^{1,3}$ and Randolf Menzel ${ }^{2,3}$ \\ ${ }^{1}$ Neuroinformatics and Theoretical Neuroscience, Institute of Biology, Freie Universität Berlin, Berlin, Germany \\ ${ }^{2}$ Neurobiology, Institute of Biology, Freie Universität Berlin, Berlin, Germany \\ ${ }^{3}$ Bernstein Center for Computational Neuroscience Berlin, Berlin, Germany \\ ${ }^{4}$ Graduate School of Life Sciences, Tohoku University, Sendai, Japan
}

\section{Edited by:}

Thomas Nowotny, University of

Sussex, UK

Reviewed by:

Roberto Fernández Galán, Case Western Reserve University, USA

Pawel Andrzej Herman, Royal

Institute of Technology, Sweden

*Correspondence:

Michael Schmuker, Neuroinformatics and Theoretical Neuroscience,

Institute of Biology, Freie Universität

Berlin, Königin-Luise-Str. 1-3, 14195

Berlin, Germany.

e-mail:m.schmuker@fu-berlin.de

${ }^{\dagger}$ Present address:

Nobuhiro Yamagata, Behavioral

Genetics, Max Planck Institute of

Neurobiology, Martinsried, Germany.
The honeybee Apis mellifera has a remarkable ability to detect and locate food sources during foraging, and to associate odor cues with food rewards. In the honeybee's olfactory system, sensory input is first processed in the antennal lobe (AL) network. Uniglomerular projection neurons (PNs) convey the sensory code from the $A L$ to higher brain regions via two parallel but anatomically distinct pathways, the lateral and the medial antenno-cerebral tract (I- and m-ACT). Neurons innervating either tract show characteristic differences in odor selectivity, concentration dependence, and representation of mixtures. It is still unknown how this differential stimulus representation is achieved within the AL network. In this contribution, we use a computational network model to demonstrate that the experimentally observed features of odor coding in PNs can be reproduced by varying lateral inhibition and gain control in an otherwise unchanged AL network. We show that odor coding in the I-ACT supports detection and accurate identification of weak odor traces at the expense of concentration sensitivity, while odor coding in the m-ACT provides the basis for the computation and following of concentration gradients but provides weaker discrimination power. Both coding strategies are mutually exclusive, which creates a tradeoff between detection accuracy and sensitivity. The development of two parallel systems may thus reflect an evolutionary solution to this problem that enables honeybees to achieve both tasks during bee foraging in their natural environment, and which could inspire the development of artificial chemosensory devices for odor-guided navigation in robots.

Keywords: dual pathway odor coding, mixture coding, antennal lobe, computational model, honeybee foraging

\section{INTRODUCTION}

Parallel olfactory subsystems are common in vertebrate and insect nervous systems. As Galizia and Rössler (2010) point out, two categories of parallel subsystems can be distinguished in the insect realm: segregate and dual parallel systems. Segregate parallel systems process different chemical stimuli (e.g., pheromone subsystems). In contrast, dual parallel systems analyze the same odorants, but with different coding and processing strategies. Often, such functional distinction is also evident in an anatomical separation.

A prominent case of a dual parallel olfactory system is found in the order of Hymenoptera, to which bees and ants belong. In these animals, uniglomerular projection neurons (PNs) relay olfactory information via two anatomically distinct tracts from the antennal lobe $(\mathrm{AL})$ to higher brain centers, that is, the mushroom body (MB) and the lateral horn ( $\mathrm{LH})$, where sensory pathways from multiple modalities converge (see Galizia and Rössler, 2010 for a review). Those two tracts are called the lateral and the medial antenno-cerebral tract ( 1 - and $\mathrm{m}$-ACT), after their anatomical location in the brain (Mobbs, 1982). These pathways are specific to the group of hymenoptera, and hence they are not present in, for example, Diptera (flies) or Orthoptera (locust; Galizia and Rössler, 2010).
In the honeybee Apis mellifera, uniglomerular PNs sending their axons along either pathway innervate segregated populations of glomeruli, the basic functional units in the AL (Abel et al., 2001). The separation of those groups of PNs on the glomerular level may indicate that the information they convey is processed separately (Galizia and Rössler, 2010). The projections of PNs from both tracts target adjacent and partially overlapping regions in the $\mathrm{MB}$ and $\mathrm{LH}$, suggesting that computational processes in those areas use information from both tracts (Müller et al., 2002; Kirschner et al., 2006).

Several studies have addressed functional differences in odor representation across the two tracts. Müller et al. (2002) reported that the segregation into two pathways is not related to the distinction between different odors or odors and pheromones, but rather appears to reflect an implementation of two different odor coding strategies. A similar observation regarding shared odor coding in both tracts has recently been reported in ants (Brandstätter and Kleineidam, 2011). This finding is supported by the observation that $\mathrm{m}$-PNs exhibited complex responses to constant odor stimuli with alternating phases of excitation and inhibition, while l-PNs preferably showed stereotypic phasic-tonic responses (Müller et al., 2002). Using the same recording technique, Krofczik 
et al. (2009) found that PNs in the l-ACT exhibited higher odor specificity, while m-ACT PNs were more broadly tuned. In addition, they found that l-ACT PNs exhibited suppressive responses to mixtures, that is, the response to the mixture was smaller than the response to the individual components. In contrast, m-ACT PNs showed hypoadditive mixture responses (mixture response resembled the strongest component response). Yamagata et al. (2009) recorded $\mathrm{Ca}^{2+}$-activity of presynaptic PN boutons in one of their target areas, the MB calyx region. They could confirm narrow vs. broad odor tuning as well as suppressive vs. hypoadditive mixture coding in 1- and m-ACTs as reported by Krofczik et al. (2009). In addition, they analyzed how the neuronal responses in both pathways depend on odor concentration. They found strong concentration dependence in $\mathrm{m}$-ACT $\mathrm{PN}$ responses in the tested concentration range, while l-ACT PN responses showed only weak concentration dependence, but responded already at very low concentrations.

Taken together, l-ACT PNs show narrower odor tuning, little concentration dependence, and suppressive mixture coding, while m-ACT PNs exhibit broader odor tuning, strong concentration dependence, and hypoadditive mixture coding (Table 1). Hence, it appears that PNs in the 1-ACT are more suited for coding the identity of an odorant but less so for representing its intensity, while PNs in the m-ACT exhibit a complementary coding strategy.

In spite of the detailed morphological and functional descriptions of the 1- and m-ACT pathways, it is still unclear how the characteristic coding strategies found therein are brought about. Lateral inhibition and gain control are well-described properties of neuronal processing in the insect AL which seem to be particularly well suited as candidate mechanisms mediating 1-ACT-like narrow odor tuning and concentration invariance. Lateral inhibition has been shown to enhance odor discriminability in the insect olfactory system (Linster and Smith, 1997; Stopfer et al., 1997; Perez-Orive et al., 2004; Wilson and Laurent, 2005). Nonuniform lateral inhibition acting between specific glomeruli has been demonstrated to improve odor discrimination in a computational model (Wick et al., 2010). Computational modeling also suggested that in honeybees, the strength of the inhibitory connection between pairs of glomeruli matches the correlation between their response spectra (Linster et al., 2005). Correlation-dependent lateral inhibition has been shown to significantly increase the performance of a machine-learning classifier framework inspired by the honeybee olfactory system (Schmuker and Schneider, 2007).

A second role of inhibition in the AL enables gain control, that is, compression of dynamic range. Gain control can be implemented by global, recurrent inhibition. Several studies have stressed that odorant discrimination can benefit from recurrent feedback inhibition (Stopfer et al., 2003; Olsen and Wilson,

Table 1 | Response properties of boutons in the I-ACT and $\mathbf{m}$-ACT pathways.

\section{I-ACT}

Concentration-invariant

Narrow odor tuning

Suppressive mixture responses

\section{m-ACT}

Concentration-dependent

Broad odor tuning

Hypoadditive mixture responses
2008; Asahina et al., 2009). Morphological and functional studies showed that in Drosophila certain populations of local inhibitory interneurons in the AL connect only specific sets of glomeruli, while other populations exhibit non-specific projections to virtually all glomeruli (Silbering et al., 2008; Chou et al., 2010; Seki et al., 2010), indicating that in Drosophila, specific lateral inhibition and global gain control are probably mediated by distinct populations of neurons, potentially belonging to two different functional subsystems. There is much less data available on local interneuron morphology in the honeybee AL, but LNs in the honeybee exhibit manifold morphology and connectivity patterns (Flanagan and Mercer, 1989; Fonta et al., 1993; Sachse and Galizia, 2002). Some LNs have been shown to target exclusively glomeruli in either the l- or m-ACT part of the AL, supporting the idea of partially separated processing of odor information across pathways (Meyer, 2011).

In this contribution, we approach the question of differential coding strategies and potential benefits in a model study. Our specific aim was to analyze whether lateral inhibition and gain control could mediate the different coding strategies that have been observed in the 1- and m-ACT pathways in the honeybee brain. To this end, we developed a computational network model that allowed us to implement a complementary odor code in dual pathways, starting from a model that is based on the concept of virtual sensors (Schmuker and Schneider, 2007). The model uses a fixed network scheme and identical neuronal resources but different parameters for the local inhibitory processing. Moreover, the presence of dual olfactory pathways suggests that they provide a substantial evolutionary advantage in the ecological niche which honeybees occupy. The model we present here allowed us to identify specific advantages of dual pathway odor coding for odor-guided navigation and foraging.

\section{MATERIALS AND METHODS CONCEPT OF THE NETWORK MODEL}

We based our network model on a previously published model for processing multidimensional data inspired by the insect olfactory system (Schmuker and Schneider, 2007). The model has no temporal component and hence neglects the fine temporal scale of odor responses. Odor-evoked activity patterns form temporal trajectories in multidimensional space which reach a stationary point shortly after stimulus onset (Stopfer et al., 2003; Galán et al., 2004; Silbering et al., 2008). Activity values in our model reflect the average activity of a neuronal population at this stationary point, and hence capture only the spatial component of olfactory coding across glomeruli (we elaborate on the limitations of this approach in Discussion).

Figure 1A outlines the connectivity of the network model. Olfactory receptor neurons (ORNs) project onto PNs and LNs which are organized in glomeruli. LNs project inhibitory connections to PNs in other glomeruli. PNs project their output to higher brain areas (not part of the model), but their output may undergo global feedback inhibition, that is, gain control.

\section{SURROGATE ORN INPUT PATTERNS}

We simulated the virtual responses pattern of ORNs to a large set of odorants as previously described (Schmuker and Schneider, 

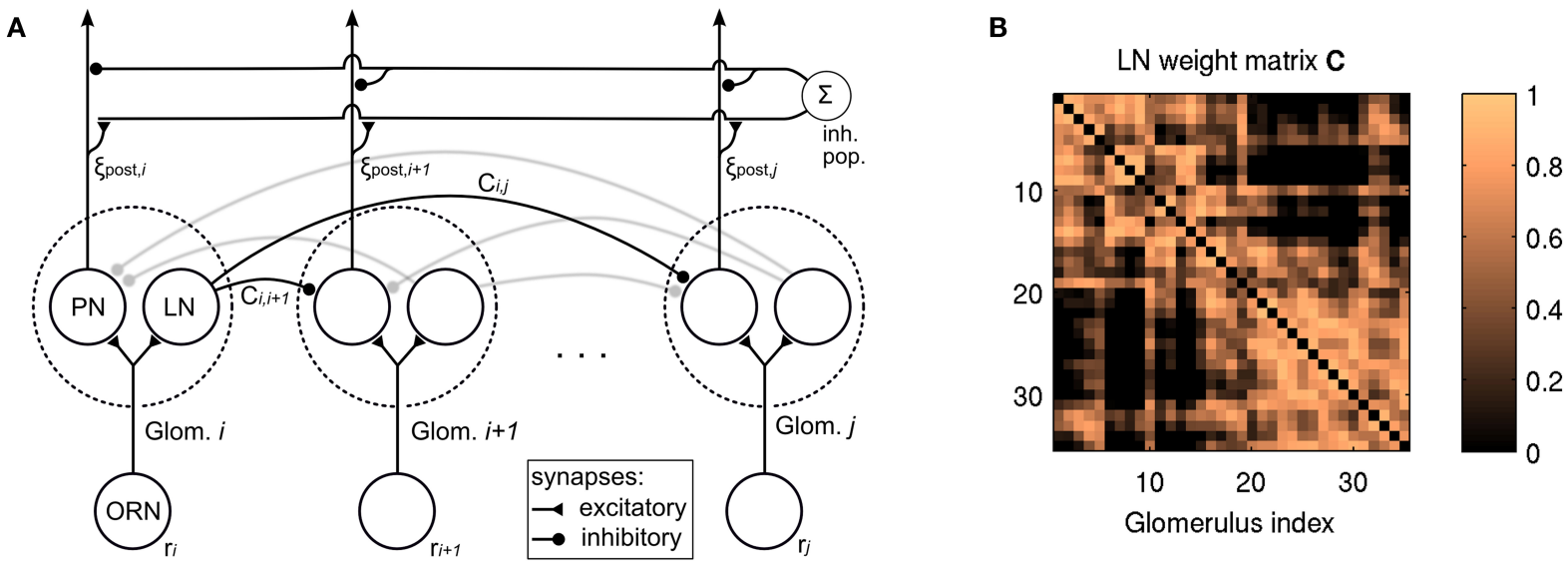

FIGURE 1 | (A) Schematic of the network topology of the model. ORN, olfactory receptor neuron; PN, projection neuron; LN, local (inhibitory) neuron. ORNs project to PNs and LNs in one glomerulus. LNs exert inhibition upon PNs in other glomeruli (Eq. 4). An inhibitory population downstream of the lateral inhibition network takes global input and exerts global inhibitory feedback (see Eqs 5 and 6). Some of the connections are shaded for better overview. (B) The weight matrix $\mathbf{C}$ used for lateral inhibition (cf. Eq. 4).
2007). Using the chemical structure of 836 odorants from the Sigma-Aldrich Flavors and Fragrances Catalog (Sigma-Aldrich, 2004) we calculated a set of 184 physico-chemical descriptors for each individual odorant using the "MOE" software package version 2005.6 (Chemical Computing Group, Montreal). Next, we trained a self-organizing map (SOM) on the 184-dimensional chemical space to mimic the evolution of the olfactory receptor repertoire using the software package SOMMER (Schmuker et al., 2006; http://sommer.sourceforge.net). Each SOM unit was then used as a "virtual receptor" to encode odorants. A virtual receptor is a point $\mathbf{p}$ in data space, and its response $r$ to an odorant $\mathbf{s}$ is determined as a function of the distance between $\mathbf{p}$ and $\mathbf{s}$. The response $r_{i}$ of the $i$ th virtual receptor - and hence the $i$ th ORN - to an odorant $\mathbf{s}$ is described by Eq. 1

$r_{i}=1-\frac{d\left(\mathbf{s}, \mathbf{p}_{i}\right)-d_{\min }}{d_{\max }-d_{\min }}$,

where $\mathbf{p}_{i}$ represents the coordinates of the $i$ th receptor, $d\left(\mathbf{s}, \mathbf{p}_{i}\right)$ denotes the city-block distance (i.e., the sum of absolute coordinate differences, Minkowski metric with $k=1), d_{\min }$ and $d_{\max }$ are the minimal and maximal distance between $\mathbf{s}$ and any $\mathbf{p}_{i}$. Hence, $r_{i}=0$ if $d\left(\mathbf{s}, \mathbf{p}_{i}\right)$ is maximal and $r_{i}=1$ if $d\left(\mathbf{s}, \mathbf{p}_{i}\right)$ is minimal. The number of virtual receptors can be adjusted by using an SOM with the desired number of units. In this study, we used a toroidal SOM layout with $5 \times 7$ units, resulting in 35 virtual receptors/ORNs. The influence of receptor count on stimulus classifiability has been studied previously (Schmuker and Schneider, 2007). Although our model can be efficiently evaluated and supports rapid exploration of large parameter spaces, we limited the model to use only 35 receptors, because increasing the number of receptor types from 35 to 96 yielded only a comparably small improvement in stimulus representation.

\section{CONCENTRATION DEPENDENCE OF PN ACTIVATION}

The previous model did not take into account odor concentration, so we needed to extend the model to support odor intensity. In a first step, we account for dynamic range of neuronal activation in the $\mathrm{PN}$ layer by expressing the presynaptic activation pattern $\xi$ of PNs by ORNs in terms of a logarithmic transfer function of receptor activation pattern $\mathbf{r}$

$\xi=\ln (\mathbf{r}+1)$,

based on experimental observations in the fruit fly where PN responses saturate with linearly increasing ORN firing (Bhandawat et al., 2007; Kazama and Wilson, 2008). Experimental evidence suggests that short term depressive synapses between ORNs and PNs provide the mechanism that underlies rate compression in the fly (Kazama and Wilson, 2008). Logarithmic transfer functions are also compatible with psychophysical observations as expressed in the Weber-Fechner law.

In a second step, concentration dependence of $\mathrm{PN}$ responses was modeled by scaling the presynaptic activation $\xi$ by the decadic logarithm of odor concentration $\Delta$,

$$
\xi_{\mathrm{conc}}=\frac{\xi}{1-\log _{10} \Delta} .
$$

We chose to represent odor concentration using this logarithmic description in order to obtain concentration values that are compatible with the physiological study which the present work was inspired from (Yamagata et al., 2009). In that study, odor concentration was reported as a dilution factor of the odorant in solvent. Dilutions ranged from $\Delta=10^{-5}$ to $\Delta=10^{0}$, and we adopted these values for the present work. In addition, this kind of scaling is compatible with physiological measurements of concentrationdependent responses in the honeybee AL (Sachse and Galizia, 2003), in which the magnitude of the glomerular response signal scaled with the logarithm of odor concentration.

\section{LATERAL INHIBITION}

Many studies have shown that lateral inhibition in the AL plays an important role in olfactory information processing (see, e.g., 
Stopfer et al., 1997; Perez-Orive et al., 2004; Wilson and Laurent, 2005). In this study, we used correlation-dependent lateral inhibition, as suggested by Linster et al. (2005) and in agreement with the previous incarnation of the model (Schmuker and Schneider, 2007).

We implemented lateral inhibition by subtracting from the activation of each PN the summed activation of all other PNs, weighted by correlation between the ORN activations over all odorants in the data set. Accordingly, we obtain the output activation pattern $\xi_{\text {post }}$ from the concentration-dependent input activation pattern $\xi_{\text {conc }}$ as

$\xi_{\text {post }}=\xi_{\text {conc }}-q \cdot \frac{C \cdot \xi_{\text {conc }}}{n}$,

where $n$ is the number of virtual receptors, $\mathbf{C}$ is the correlation matrix with $C_{i, j}$ containing the Pearson correlation coefficient for the responses of the $i$ th and $j$ th ORN, and $q$ is a scaling factor which allows to adjust the overall strength of inhibition. Elements on the diagonal as well as negative entries of $\mathbf{C}$ were set to zero to avoid self-inhibition and to reflect the fact that inhibited inhibitory interneurons have no post-synaptic effect. Figure 1B depicts the weight matrix $\mathbf{C}$. It is calculated on the basis of receptor responses to all single odorants in the data set and thus reflects the similarity structure in the input space. It is constant throughout this study. Note that the structure of lateral inhibition is controlled by $\mathbf{C}$, but $q$ is factored out to allow control of the overall strength of inhibition. The factor $n$ was introduced in the previous work to enable comparison of $q$ values over different receptor counts. Although $n$ is constant in this study, we kept it as a parameter for consistency with previous work.

\section{FEEDBACK INHIBITION}

Our implementation of feedback inhibition was motivated by two experimental observations. First, it has been shown that 1-ACT boutons respond to very weak odor concentrations, while m-ACT PN boutons do not (Yamagata et al., 2009), meaning that PNs in the l-ACT react more sensitive to weak stimuli compared to $\mathrm{m}$ ACT PNs. Second, in the honeybee, the input to l-ACT PNs in the AL exhibits strong dependence on odor concentration. This has been demonstrated in a study which measured the $\mathrm{Ca}^{2+}$ signal in backfilled PNs in the AL, where dendritic $\mathrm{Ca}^{2+}$ activity likely constituted the majority of the signal (Sachse and Galizia, 2003). It hence follows that gain control in the honeybee AL must act downstream of PN dendrites, but upstream of PN boutons.

In order to capture this behavior, we extended the model with a two-step gain control mechanism consisting of a sensitivity boost and subsequent feedback inhibition. Our implementation of gain control is described as

$\boldsymbol{\xi}_{\mathrm{out}}=\frac{\boldsymbol{\xi}_{\mathrm{post}} \cdot \beta}{\rho}$

where $\beta$ denotes the sensitivity boost factor, and $\rho \geq 1$ describes the activity of a population of inhibitory neurons that mediates global feedback inhibition on PN output (see Figure 1A) in a divisive manner. The inhibitory population receives input from all PNs; its activity is described as

$\rho=\left\{\begin{array}{cc}1, & \text { if }\left|\boldsymbol{\xi}_{\text {post }}\right| \leqslant \theta \\ \frac{\left|\boldsymbol{\xi}_{\text {post }}\right|}{\theta}, & \text { if }\left|\boldsymbol{\xi}_{\text {post }}\right|>\theta\end{array}\right.$.

$\left|\boldsymbol{\xi}_{\text {post }}\right|$ denotes the L1-norm of the PN output pattern (the sum of the activity of all PNs), $\theta$ the (non-zero) activation threshold of the inhibitory neurons. The case of $\rho \equiv 1$ describes the case where no feedback inhibition is present in the network. The inhibitory population responds as total $\mathrm{PN}$ output exceeds $\theta$, which we set to the average level of PN output for all patterns in our data set without lateral inhibition and gain control. We normalized the amount of inhibition by $\theta$, so that $\rho \approx 1$ when $\left|\xi_{\text {post }}\right| \approx \theta$. Taken together, gain control in our model globally boosts the PN signals if summed activity is weak and attenuates them if it the summed response is strong.

In our physiological observations, the output from m-ACT neurons at the highest concentration was in the same range as the response from l-ACT neurons over concentrations (Yamagata et al., 2009). Hence, we set $\beta$ to 6 throughout this study, compensating the concentration discount for the lowest concentration $\left(10^{-5}\right)$ in Eq. 3. Thus, when gain control is effective, the amplitude of an input pattern at the lowest concentration matches the amplitude of the same pattern at the highest concentration without gain control.

\section{RESULTS}

Stimulus representation differs in a characteristic way across two parallel pathways in the honeybee olfactory system (Table 1; Yamagata et al., 2009). Our aim was to identify network properties underlying the observed differential odor coding, and to investigate the potential benefit of such a dual coding strategy. To this end, we used a computational network inspired by the neural circuits in the olfactory system of the honeybee, based on a model of olfactory processing in the AL (Schmuker and Schneider, 2007). We investigated the roles which glomerulus-specific lateral inhibition and non-specific feedback inhibition ("gain control") may play in creating the distinct coding properties in the l- and m-ACT. Since comprehensive characterizations of the molecular receptive fields of the honeybee's ORNs are not available, we used surrogate data as input to the AL (see Materials and Methods for details).

\section{EFFECT OF LATERAL INHIBITION STRENGTH AND GAIN CONTROL ON THE WIDTH OF ODOR TUNING CURVES}

It has long been known that lateral inhibition effects contrast enhancement and supports a sparse stimulus code in sensory processing of various modalities. In the honeybee, PN boutons in the 1-ACT pathway respond to fewer odorants and thus exhibit narrower odor tuning profiles than their m-ACT counterparts - in other words, 1-ACT PNs exhibit a sparser odor tuning code (Yamagata et al., 2009). In our model, increasing lateral inhibition in the absence of gain control leads to an overall reduction in activity, likely making it more difficult for downstream neurons to detect the response patterns (Figure 2A, upper row). Obviously, gain 
control (Eqs. 5 and 6) can act as a mechanism to recover the activity pattern (Figure 2A, lower row). With gain control enabled, the activity pattern retains its strength, even when lateral inhibition is strong $(q>1.0)$. At the same time, the sparsening effect of lateral inhibition can be observed: many PNs respond when the lateral inhibition is weak ( $q$ is low), but when $q$ increases the activity pattern gets sparser and its contrast is enhanced.

We then tested whether lateral inhibition as described by Eq. 4 can account for narrow odor response profiles and sparse odor coding in 1-ACT PNs. Figure 2B illustrates the tuning profile of a single PN with respect to the entire set of 836 odors in our database. Without gain control, the odorant response profiles show overall reduced response magnitude, but their shapes are not changed (Figure 2B, left panel). But, with gain control in effect, odorant tuning curves become narrower as lateral inhibition is increased (Figure 2B, right panel). Thus, we could achieve substantial narrowing of the odorant response profiles only when gain control was in effect and, at the same time, lateral inhibition was strong.

One might argue whether increasing input gain alone may also be sufficient to produce narrower tuning curves in presence of strong lateral inhibition. We tested this hypothesis by increasing sensitivity alone without applying feedback inhibition $(\beta=6$ and $\rho=1$ in Eq. 6). The resulting tuning curves did not become narrower, only their amplitude was increased (Figure 2C). This observation further supports that both, increased input sensitivity and gain control by feedback inhibition are required to reproduce narrow odor tuning when lateral inhibition is strong.

Lateral inhibition affects the discriminability of odorant representations in PNs. We used the Euclidean distance between PN response patterns as an estimate for their discriminability. Distance was calculated for each pair of odors, so that we ended up with $836^{*} 835^{*} 0.5=349,030$ distance values for each value of lateral inhibition strength $q$. Two observations can be made from the distribution of distances (Figure 3). First, when gain control was in effect, increasing the strength of lateral inhibition also caused the distance between odorant representations to increase. Second, when there was no gain control, the distance between odorant representations slightly decreased with increasing lateral inhibition. This observation is a consequence of the decreasing overall amplitude of response patterns with increasing lateral inhibition. This result indicates that both mechanisms, gain control and lateral inhibition, need to be combined to achieve improved pattern separability, and hence discriminability by downstream neurons.

This interpretation relies on the assumption that neuronal responses are not invariant to scaling, that is, the assumption that small differences in the signal are more difficult to discern than large differences. Assuming that stochastic fluctuations in the stimulus, the transduction chain, synaptic release, external conditions, etc., do not or only weakly scale with signal amplitude, they will affect weak signals more than strong ones. In consequence, large differences in activity between PNs will be more reliably detected
A
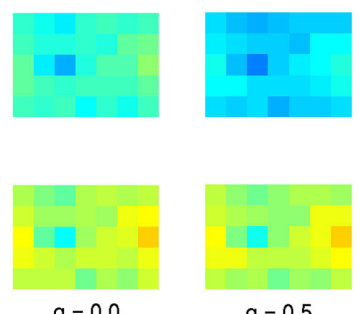

$q=0.5$

B

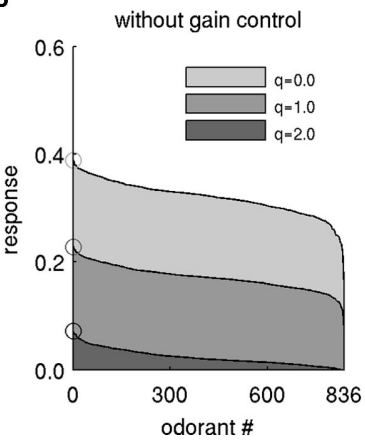

without gain control

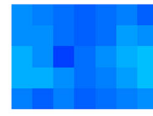

with gain control

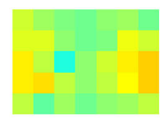

$q=1.0$
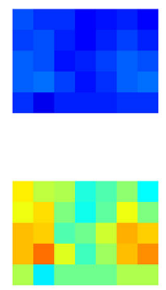

$q=1.5$

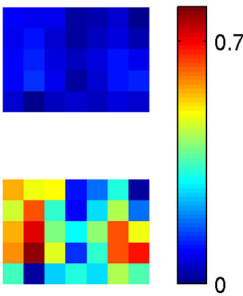

$q=2.0$ 0.7

C
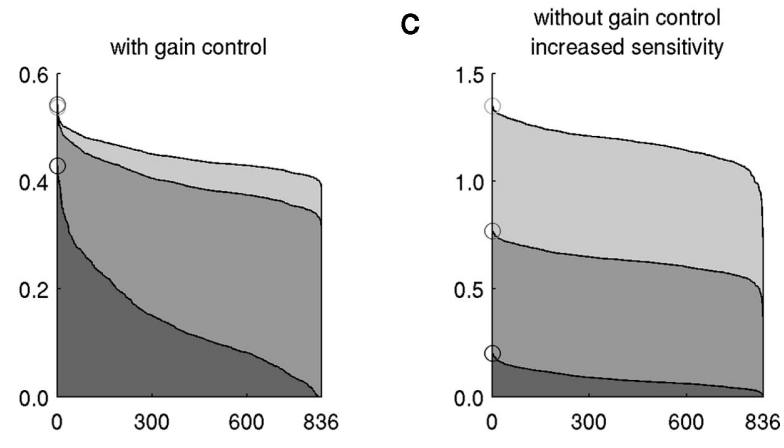

FIGURE 2 | Effect of lateral inhibition on PN response profiles. (A) The pattern evoked by a single odor (butyl proprionate, modeled concentration $10^{-1}$ ) transformed by lateral inhibition of different strengths, indicated by the factor $q$. Each square in the $5 \times 7$ grid corresponds to the $P N$ response within one model glomerulus. (B) Response magnitude in PN no. 17 (fourth column, second row) for all 836 odors for three levels of lateral inhibition, without (left) and with gain control and increased sensitivity ( $\beta=6$, right). The odors are arranged along the abscissa with descending response strength such that the strongest odorant is always displayed on the left. Circles denote the magnitude of the leftmost odorant. (C) Same as (B) without gain control, but with PN sensitivity increased by the same factor $(\beta=6)$. Note the different scale of the ordinate. 


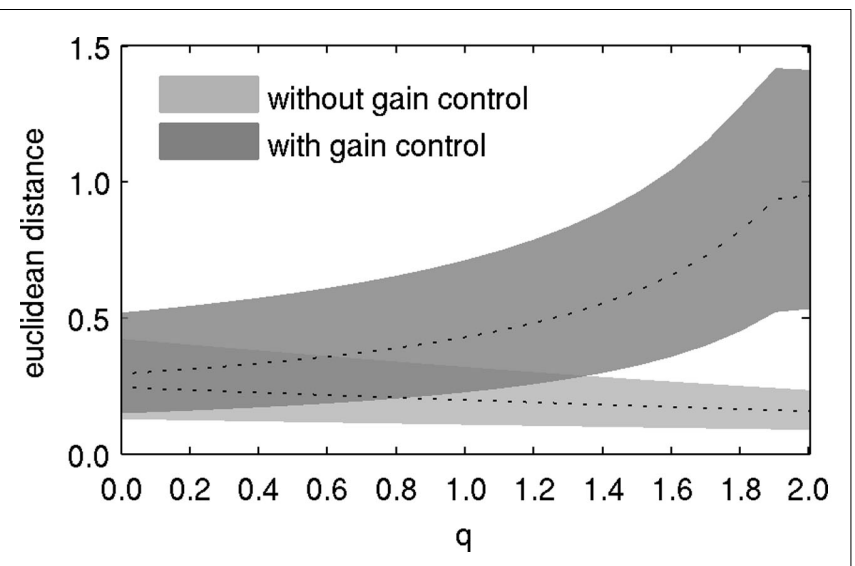

FIGURE 3 | Discriminability of odor representations in PNs, as indicated by the average pairwise euclidean distance between PN response patterns to all odorants. Dotted lines denote the median, shaded areas denote the difference between the 10th and the 90th percentile of the distribution of distances.

than weak differences. To demonstrate this aspect would require a more elaborate model which is outside the scope of this study.

Taken together, lateral inhibition alone leads to reduced responses without substantial sharpening of PN tuning curves. It only improves odor discrimination together with gain control in the scope of our model.

\section{REPRODUCTION OF PHYSIOLOGICAL OBSERVATIONS}

We wondered whether we could also reproduce the characteristic differences in odor concentration and mixture representation across 1 - and $\mathrm{m}$-ACT that have been described in physiological studies (Table 1). In the following, we describe the extent to which our model is able to reproduce physiological observations regarding representation of odor concentration and mixtures of odors. Since we implemented gain control and lateral inhibition as separate mechanisms, we were able to gauge their effects on odor coding separately.

In our previous physiological experiments, we observed positive concentration dependence in m-ACT PNs and no or even negative concentration dependence in l-ACT PNs (Yamagata et al., 2009). We first wanted to investigate to what extent gain control alone could reproduce our experimental observations on concentration and mixture coding, when there is no lateral inhibition involved ( $q=0$ in Eq. 4). Without gain control, the PN responses naturally exhibited strong concentration dependence, matching the behavior of m-ACT PNs (upper row in Figure 4A). With gain control, this dependence was virtually eliminated (lower row in Figure 4A). In many cases we even observed negative concentration dependence, as for example in the upper trace in Figure 4B. Negative concentration dependence occurs when a PN is strongly activated by its input, but gets attenuated through feedback inhibition as the other PNs increase their responses with rising concentration. It was weak in magnitude in the cases where it was present. Negative concentration dependence was also observed physiologically in a large fraction of l-ACT PN boutons (cf. Figure 4 in Yamagata et al., 2009).
In order to depict the general effect of feedback inhibition on intensity representation, we followed the approach of our physiological study by estimating the representation of different odor concentrations in individual PNs. To this end, we performed linear regression of the concentration response curves (Figure 4B) for the response of each of the $35 \mathrm{PNs}$ to each of the 836 odors, obtaining $836 \cdot 35=29,260$ slopes. We then compared the distribution of slopes for the two conditions with and without gain control (Figure 4C). Without gain control the regression lines had strictly positive slopes, but when gain control was enabled, the regression slopes took small values close to zero, both in the positive and negative range. Hence, the strong concentration dependence observed in m-ACT PNs was reproduced when gain control was disabled, while l-ACT-like concentration coding could be achieved with gain control through feedback inhibition.

Responses of 1-ACT PNs to mixtures of two odorants have been described to be weaker than their responses to each of the components (suppressive mixture coding), while in m-ACT PNs mixture responses have been observed to be as strong or stronger than the response to each component (hypoadditive mixture coding; Krofczik et al., 2009; Yamagata et al., 2009). We systematically varied the strength of lateral inhibition in absence and in presence of gain control to investigate the role of those network properties in reproducing characteristic differences in mixture coding across pathways. To this end, we implemented mixtures of two odorants as linear superposition of their patterns (Eq. 7)

$\xi_{\text {mix }}=\ln \left(\mathbf{r}_{\mathrm{A}}+\mathbf{r}_{B}+1\right)$,

with $\mathbf{r}_{\mathrm{A}}$ and $\mathbf{r}_{\mathrm{B}}$ the receptor patterns of the two components $\mathrm{A}$ and $\mathrm{B}$, and $\boldsymbol{\xi}_{\text {mix }}$ the activity pattern in response to the mixture. As in Eq. 2, we used a logarithmic transfer function to account for the dynamic range of neuronal activation. Activity patterns are combined before applying the logarithmic transfer function which models PN activation. In the biological scope, this approach corresponds to linearly adding the responses on the receptor level, which is in line with physiological findings in various organisms. For example, Tabor et al. (2004) observed in zebrafish that activity patterns evoked by odor mixtures in afferents to the olfactory bulb could be predicted from the component patterns, suggesting that mixture interactions in the peripheral olfactory system exhibit only weak non-linearity. Similar findings have been described in Drosophila (Silbering and Galizia, 2007), the moth Spodoptera litoralis (Carlsson et al., 2007), and the honeybee (Deisig et al., 2006). To illustrate the effect of mixing odorants, we depict response patterns of two odorants (acetaldehyde and butyl proprionate) and their mixture without gain control in Figure 5A. We used the (virtual) concentration of $10^{-1}$, the same concentration that we used in the mixture experiments in our experimental study (Yamagata et al., 2009). PNs exhibited hypoadditive mixture coding, as the response of each PN to the mixture was higher than to each of the components, although the mixture response is not a strictly additive superposition of both components because of the PN's logarithmic transfer function (Eq. 7).

We then checked the influence of gain control and lateral inhibition on mixture coding. Figure 5B shows the response patterns 


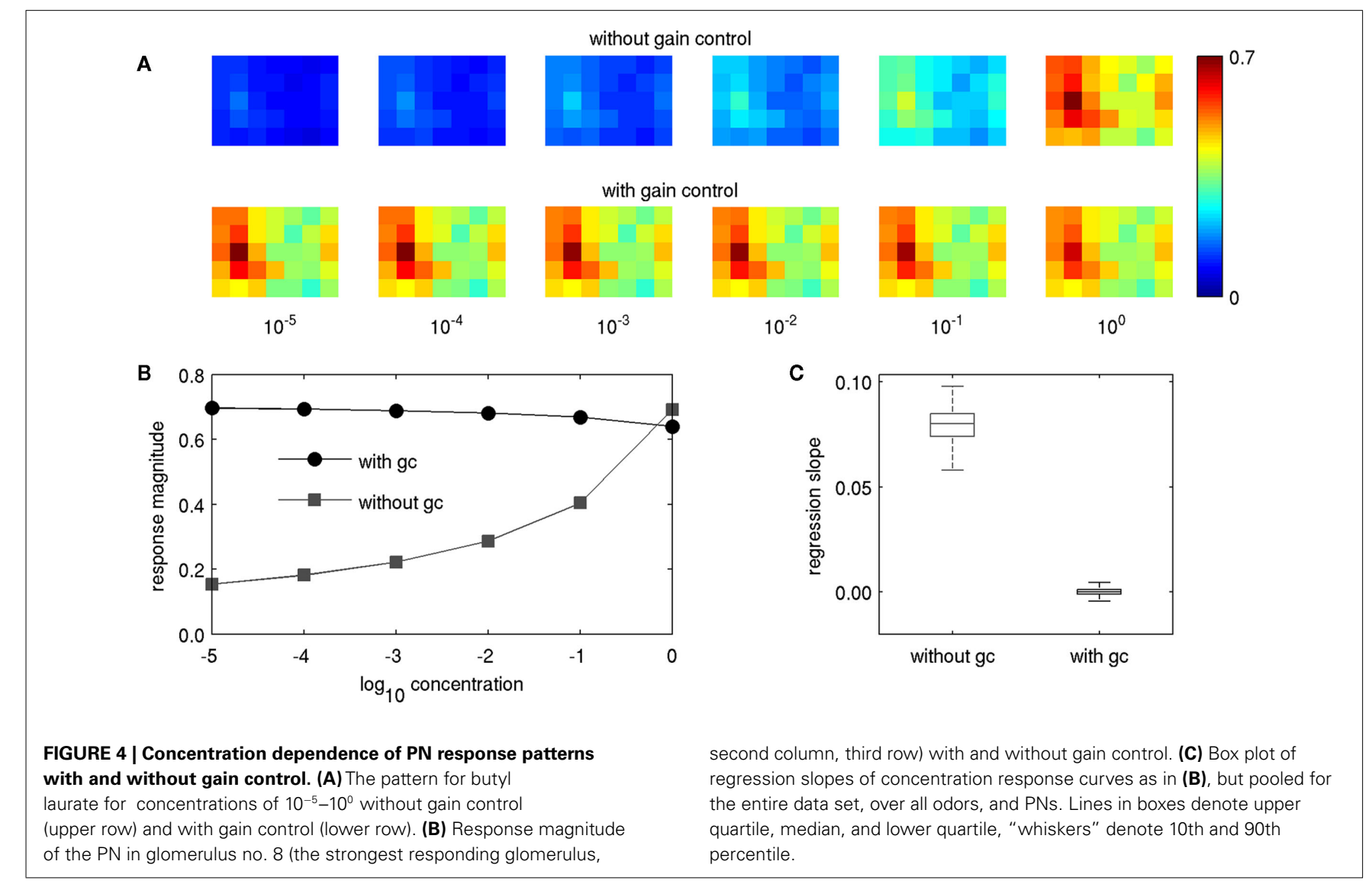

for components and mixtures with gain control, and for three different levels of lateral inhibition (controlled by the factor $q$ ). Gain control generally promoted suppressive mixture coding. Lateral inhibition led to increased contrast of the components as well as the mixtures (cf. Figure 2), but it was difficult to discern an effect on mixture coding for $q=1$. A clear effect became visible for $q=2$, but it is difficult to estimate from the visualization of the response pattern alone whether this condition supported suppression or hypoadditivity. A significant portion of PNs were silenced by strong lateral inhibition for $q=2$, resulting in sparse activity in the component-evoked pattern, and hence lower overall gain. With lower total gain, gain control became less effective (see Eq. 6), and its suppressive influence on mixture representation became smaller. This observation indicates that values of $q \geq 2$ represent an extreme case and are most likely not useful in reproducing experimental observations.

We wanted to obtain a quantitative assessment of whether PNs express suppressive or hypoadditive responses to a mixture of two components $\mathrm{A}$ and $\mathrm{B}$ over a range of parameters. To this end, we calculated for each PN the index for mixture additivity $\kappa$ (Krofczik et al., 2009; Eq. 8)

$\kappa=\frac{\xi_{i, \operatorname{mix}}-\max \left(\xi_{i, \mathrm{comp} A}, \xi_{i, \text { comp B }}\right)}{\xi_{i, \operatorname{mix}}+\max \left(\xi_{i, \mathrm{comp} A}, \xi_{i, \text { comp B }}\right)}$,

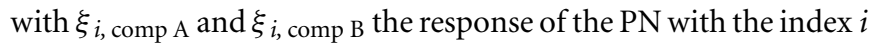
to the component $\mathrm{A}$ and $\mathrm{B}$, respectively, and $\xi_{i \text {, mix }}$ the response of
PN $i$ to the mixture. Hence, values of $\kappa>0$ indicate hypoadditive mixture representation, while values of $\kappa<0$ indicate suppressive mixture representation. Figure 5C shows the distribution of $\kappa$ across PNs for the mixture from Figure $5 \mathbf{A}$, with and without gain control, for $q$ in the range between zero and two. Gain control supported suppressive mixture representation $(\kappa<0)$ over a large range of $q$. Although the values are still close to zero, the median as well as the 10th and 90th percentiles of the distribution of $\kappa$ were below zero, indicating a clear trend to suppressive responses. In contrast, mixtures were represented in a hypoadditive way $(\kappa>0)$ when there was no gain control. Strikingly, the amount of lateral inhibition had virtually no effect on the mixture responses being hypoadditive or suppressive over a large range of inhibition strength. That stereotypic behavior began to break down only as $q$ approached the extreme value of 2 , when no clear trend toward hypoadditive or suppressive mixture coding could be discerned anymore, confirming our qualitative observations from Figure 5B. Hence, the presence of gain control appears to be the dominant factor mediating the characteristics of mixture coding over a large range of parameter values.

Natural odors are typically blends of many different components (Knudsen et al., 2006; see Raguso, 2008 for a review), and the ability to discriminate blends is important for foraging (Wright et al., 2002). Honeybees can distinguish fine differences in blend composition foraging (Wright et al., 2005), and it has shown that AL processing plays an important role in mixture discrimination (Deisig et al., 2010). As lateral inhibition and gain control enhance 


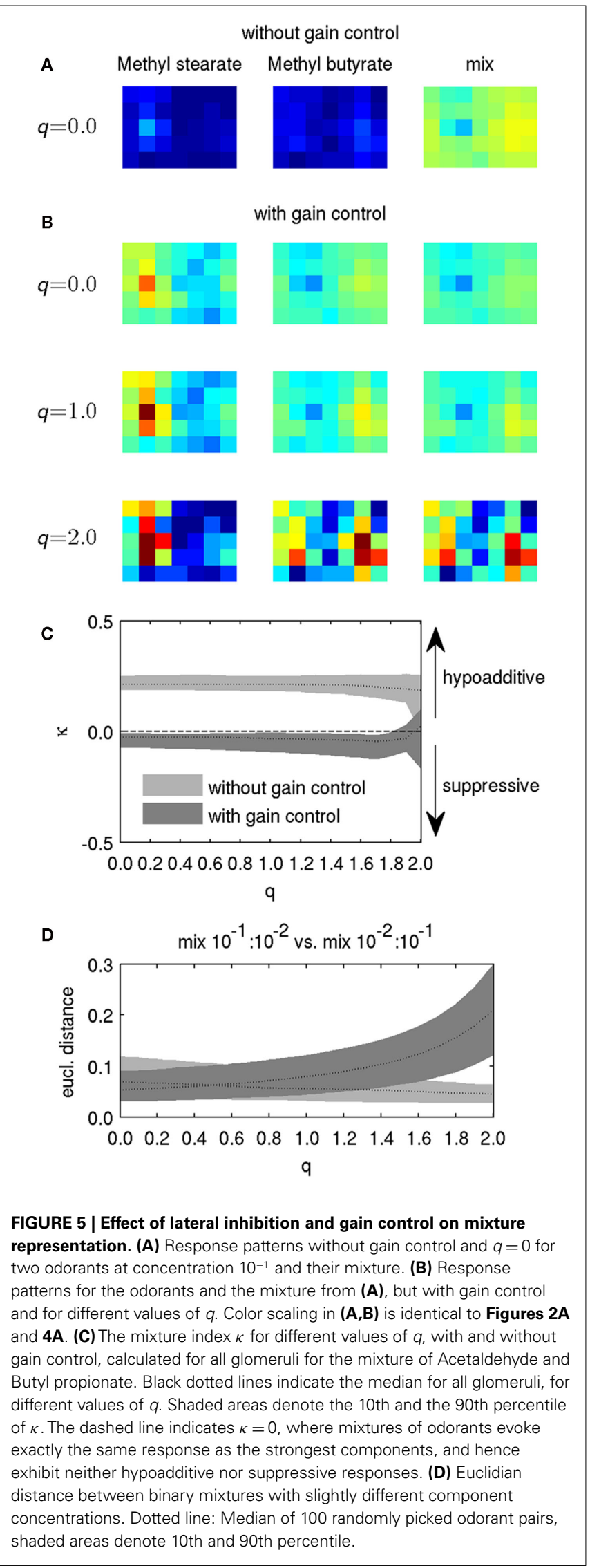

pattern discriminability (cf. Figure 3), we hypothesized that these mechanisms also affect mixture discrimination. We tested whether our model reproduces this observation by calculating the distance between artificial binary blends with slight differences in the proportion of components. To this end, we randomly picked odorant pairs from our data set and created two binary mixtures from each pair that differed slightly in their component ratio. In one mixture the ratio of concentration between odor $\mathrm{A}$ and odor $\mathrm{B}$ was $\mathrm{A}: \mathrm{B}=10^{-1}: 10^{-2}$, while the ratio was reversed in the other mixture $\left(\mathrm{A}: \mathrm{B}=10^{-2}: 10^{-1}\right)$. We calculated the Euclidean distance between the response patterns evoked by the two mixtures after processing them with various levels of lateral inhibition. As with single odors, discriminability of mixtures was enhanced for high levels of lateral inhibition in combination with gain control (Figure 5D), thus favoring the discrimination of weak differences in blends of odorants.

Taken together, lateral inhibition enhanced discriminability of mixtures on the level of PN response patterns, but it hardly affected whether mixtures were represented in an additive or subtractive way at single PNs. The latter aspect of mixture coding was mediated mainly by the presence or absence of gain control, which is required to avoid that the signal gets lost when lateral inhibition is strong.

\section{MODEL-BASED EXPLANATION OF AN ATYPICAL EXPERIMENTAL OBSERVATION}

The mechanism we suggest here helps to understand an important detail in our previous experimental observations on PN boutons in the MB. There was one odor, hexanal, which exhibited concentration dependence more in l-ACT PN boutons than in mACT boutons, and was more sparsely represented in m-ACT PN boutons than in l-ACT PN boutons, hence exhibiting "inversed" behavior (cf. Figure S3 in Yamagata et al., 2009). Both effects can be explained in a straightforward manner from our model, considering the particular response pattern that hexanal evokes in the AL. Hexanal activates comparably few glomeruli in the part of the 1-ACT that is accessible to imaging (Sachse et al., 1999). In the mACT, sparse input will lead to sparse output, but in the l-ACT, PNs in glomeruli which receive only weak excitatory input may exhibit stronger responses at their output through the sensitivity boost that gain control implies (factor $\beta$ in Eq. 5), apparently reducing sparseness compared to m-ACT PNs. This explains why hexanal is sparsely represented in m-ACT PN boutons, but exhibits less sparse representation in l-ACT PNs.

Along a similar line of thought, our model explains the other effect we observed, namely concentration-dependent representation of hexanal in otherwise concentration-insensitive l-ACT boutons. In the l-ACT, gain control will not exert much influence when only few PNs are activated, as the strength of gain control depends on the sum of activation in all PNs (Eq. 6). So gain control is less effective for odors which activate few PNs (like hexanal), and in consequence those odors will exhibit concentration dependence also with l-ACT-like network parameters.

\section{DISCUSSION}

In the present study, we have analyzed putative network mechanisms which may generate the characteristic features of odor 
Table 2 | Network properties reproducing I-ACT and m-ACT-like coding characteristics and their functional significance.

\begin{tabular}{lr}
\hline I-ACT & m-ACT \\
\hline $\begin{array}{l}\text { Strong lateral inhibition } \\
\text { good odor discrimination }\end{array}$ & Weak lateral inhibition \\
$\begin{array}{l}\text { Strong gain control } \\
\text { weak concentration discrimination odor discrimination }\end{array}$ & Weak gain control \\
\end{tabular}

coding that have been described across two parallel pathways in the honeybee olfactory system. We have demonstrated that the complementary features of odor coding at the level of the MB input found across the two pathways (Table 1) can be captured in a single model network by using different parameter settings (Table 2). We could reproduce coding properties observed in PNs of the l-ACT, namely narrow odor tuning, concentration invariance, and suppressive mixture coding by strong lateral inhibition and strong gain control in the model network. On the contrary, weak lateral inhibition and absence of gain control reproduced coding properties of PNs in the m-ACT, namely broad odor tuning, concentrationdependent responses, and hypoadditive mixture representation. As a result in the biological scope, this finding is compatible with the assumption that in the honeybee $\mathrm{AL}$, the $\mathrm{l}-\mathrm{ACT} / \mathrm{m}$-ACT pathways represent two partly segregated subnetworks where parameters are tuned to differential functional properties using the same neuronal ground plan. This constrains current hypotheses on the connectivity within and between both pathways (see Galizia and Rössler, 2010 for a review). Our results also suggest dual pathway odor coding may be of particular value in odor-guided foraging, when precise information about stimulus intensity and identity is required simultaneously.

Several assumptions underlying our model are vital for the validity of these interpretations. For the present study we assumed that odor selectivity is similar for ORNs that innervate glomeruli of the l-ACT and those innervating m-ACT. Also, we assumed homogenous odor sensitivity in ORNs that innervate glomeruli of either tract. In our model, tract-specific differences in odor sensitivity arise only at the level of PNs and result from computations within the AL network and (potentially) feedback from downstream neuron populations (see below). To our best knowledge, there is currently no experimental evidence that contradicts any of these assumptions.

\section{CANDIDATE NEURON POPULATIONS IN THE HONEYBEE BRAIN MEDIATING FEEDBACK INHIBITION ON THE AL OUTPUT}

Our modeling results suggest that gain control is important to explain the coding differences between 1 -ACT and $\mathrm{m}$-ACT. In order to test this hypothesis in future studies, it is important to know which neuron populations could mediate the gain-controlling feedback inhibition in the honeybee olfactory pathway. Evidence from morphological and physiological studies in the honeybee brain suggests three possibilities.

The first possibility is that gain control is provided by inhibitory feedback neurons in the protocerebro-calycal tract (PCT). PCT neurons have post-synaptic profiles in the alpha-lobe of the $\mathrm{MB}$ and project up to the calyx region (Grünewald, 1999; Okada et al.,
2007). In the MB calyx, PCT neurons form reciprocal connections to PN boutons within so-called microglomeruli (Ganeshina and Menzel, 2001). At least a part of the PCT neurons are GABAergic. They could mediate the observed feedback inhibition since the physiological observations which we partly ground our model upon are based on measurements of presynaptic PN boutons within these microglomeruli (Yamagata et al., 2009). Dendrites of PCT neurons span large areas in the alpha-lobe, where they can pick up the population response from a large fraction of Kenyon cells (KCs), which in turn may reflect the overall input of PNs to the MB. Hence, the connection PN-KC-PCT-PN may form an inhibitory feedback loop. Recently, a similar mechanisms has been proposed in locust, where negative feedback from the MB output back to its input is mediated by a large inhibitory neuron (Papadopoulou et al., 2011), although it is unclear whether that neuron targets only KC dendrites or if it also synapses onto PN axonal boutons.

The second possibility is that feedback inhibition from PCT neurons is mediated directly through the widespread axodendritic ramifications that these neurons form in the calyx region. Within microglomeruli, PCT neurons can pick up the excitatory input directly from PNs and feed it back on the boutons through their inhibitory synapses in the entire calyx region (Ganeshina and Menzel, 2001; Hourcade et al., 2010). However, it is unclear how signals traveling on the axo-dendritic structures between microglomeruli would interfere with action potentials arriving from the alpha-lobe.

As a third possibility, gain control could be exerted by local inhibitory interneurons in the AL targeting the PN dendrites very close to the integrating segment, that is, in the core region of glomeruli (Fonta et al., 1993). The dendrites would still be showing concentration-dependent responses, but due to hyperpolarization close to the integrating segment, fewer action potentials might be generated. Hence, $\mathrm{Ca}^{2+}$-imaging of backfilled PNs in the $\mathrm{AL}$ would still show concentration-dependent responses, in line with experimental observations (Sachse and Galizia, 2003). At the same time, this arrangement would be compatible with the absence of concentration dependence in l-ACT PN output boutons in the MB that we observed (Yamagata et al., 2009).

The question whether gain control in l-ACT PNs is achieved already in the AL or only at the level of the PN boutons may be resolved by measuring the activity in axons of 1-ACT PNs, for example by measuring electrophysiological signals extracellularly in the tract (Brill et al., 2011). Also, more detailed physiological and morphological characterizations of local interneurons in the honeybee AL are required (Meyer, 2011; Meyer and Galizia, 2011).

\section{POTENTIAL RELEVANCE OF DIFFERENTIAL ODOR CODING IN ODOR-GUIDED NAVIGATION AND FORAGING}

In our model, lateral inhibition and gain control together can enhance discriminability of single odors (Figure 3) and blends (Figure 5D). On the other hand, gain control inevitably leads to a loss of intensity information (Figure 4). Hence, there exists a tradeoff between discriminability of odors and representation of their intensity. The co-existence of a concentration-invariant and highly discriminative coding scheme in the l-ACT and a concentrationsensitive, less discriminant coding scheme in the m-ACT might 
indicate an evolutionary adaptation of the honeybee olfactory system to achieve both, concentration sensitivity and accurate detection of odors even at low concentration.

Both capabilities are of vital importance for odor-guided foraging. For example, a foraging bee may encounter a faint odor trace emanating from a distant flower. Under these conditions, detection and identification of the odorant requires high sensitivity at low odor concentrations and narrow odor tuning, as found in lACT PNs. If the odor is attractive, the bee will try to approach the odor source. Information about the distance to the source is encoded in odor plumes in two factors: odor intermittency, that is, the frequency at which the odor is encountered, and odor concentration (see Riffell et al., 2008 for a review). Although it has been shown that odor intermittency alone can be used to successfully approach the source of a pheromone via "infotaxis" (Vickers, 2000; Vergassola et al., 2007; Moraud and Martinez, 2010), it is clear that the distance to the source of an odor is also encoded in its concentration within plume filaments (Murlis, 1997; Thistle et al., 2004; Zollner et al., 2004) and hence odor concentration is a powerful cue for distance to the source. Information about concentration is encoded in m-ACT PNs. Thus, by integrating the information about odor identity from 1-ACT PNs and concentration from m-ACT PNs, the honeybee may be able to detect and approach an odor source more efficiently than when relying on infotaxis alone. In addition, odor concentration can be very high in close vicinity to the odor source, e.g., at the blossom of a flower. Odor discrimination (and hence odor learning) in this scenario requires that the $\mathrm{PN}$ response is not saturated. Gain control may counteract saturation of 1-ACT PNs, enabling reliable, and concentration-invariant encoding of odor identity even at high concentrations. Interestingly, previous model studies have shown that, indeed, gain control can improve learning of odor identity (Huerta et al., 2004; Nowotny et al., 2005).

If our hypothesis is correct we may predict that a honeybee whose l-ACT is dysfunctional will still be able to approach odor sources, but it should exhibit severe impairments in general odor discrimination, in odor learning at high concentrations, and in odor detection at low concentrations. On the other hand, a honeybee lacking a functional $\mathrm{m}$-ACT should exhibit little impairment in odor detection and discrimination, but it may have an impaired ability to approach an odor source, unless the lack of intensity information conveyed by $\mathrm{m}$-ACT PNs is compensated by another mechanism or other neurons, like for example ml-ACT PNs or multi glomerular PNs (see Galizia and Rössler, 2010 for detailed overview on PN types in the honeybee $\mathrm{AL}$ ).

The latter point leads to the question why an entire odor coding system like the m-ACT should be used to represent intensity if in principle only one channel would be necessary. However, in an odor rich environment, where one odor needs to be identified out of many odors, and its concentration to be determined, it may be important to have not only a plain intensity signal, but also substantial odor information associated with it. This information may help to bind the concentration information relayed by the m-ACT to the odor identity information relayed by the 1-ACT in higher brain regions where both pathways converge. Additional experimental and theoretical studies are needed to test this hypothesis.

\section{RELATION TO ANOTHER PROPOSED MECHANISM FOR CODING ODOR IDENTITY VS. INTENSITY}

Stopfer et al. (2003) found that in locust, odor intensity, and identity are represented in parallel within the same spike train, encoded in temporal patterns which change dynamically during odor presentation. Locusts do not have dual olfactory pathways like the l- and $\mathrm{m}$-ACT in honeybees, so the challenge of encoding identity and intensity at the same time may be solved differently in these animals. Nevertheless, the presence of parallel pathways for intensity and identity in the honeybee raises the question of the particular benefit of that dual system. One potential advantage of having a parallel representation of those stimulus features as opposed to encoding it in temporal features is that in a parallel system, identity and intensity can be provided almost immediately after stimulus onset. If those features have to be decoded from a spatio-temporal pattern, a certain amount of time is required until that pattern has evolved to encode a sufficient amount of information. The olfactory system of honeybees signals the presence of behaviorally relevant odors with very little delay in conditioning experiments (Strube-Bloss et al., 2011). In those experiments, MB-extrinsic neurons which encode the value of the stimulus (rewarded vs. non-rewarded) exhibited an odor-driven population response $60 \mathrm{~ms}$ after odor onset, which is approximately $20 \mathrm{~ms}$ after a specific representation of the odor has built up in the PN ensemble in the AL (Krofczik et al., 2009). Moreover, the representation of the rewarded odor at the MB output became significantly different from unrewarded stimuli as quickly as $140 \mathrm{~ms}$ after odor onset. These findings suggest that the olfactory system of the honeybee can indeed benefit from a rapid encoding of odors in the input, and hence from parallel pathways. Moreover, in a natural environment odor plumes are encountered intermittently and concentration is fluctuating very fast when crossing a filament (Murlis, 1997; Thistle et al., 2004; Zollner et al., 2004; Riffell et al., 2008). If odorant concentration and identity are to be linked, it is hence important to measure the maximum odor concentration with high temporal precision. This capability may particularly facilitate odor-guided navigation in an odor rich environment where the animal encounters plumes from different odor sources and needs to distinguish between them.

\section{LIMITATIONS OF THE PROPOSED MODEL IN THE BIOLOGICAL SCOPE}

The network model presented in this study considers only the spatial component of the olfactory code and ignores detailed response dynamics. The main reason for this restriction is that our model is partly based on experimental results obtained from $\mathrm{Ca}^{2+}$ imaging of $\mathrm{PN}$ boutons, which provides only poor estimates of the temporal dynamics of the neural code. However, there is much evidence for the relevance of temporal aspects of the neural code. Stopfer et al. (1997) have demonstrated the importance of odor-evoked oscillations in neuronal activity in the AL for odor discrimination in the honeybee. Krofczik et al. (2009) suggested that PNs may employ a latency code for representing odors, where the temporal delay between stimulus onset and $\mathrm{PN}$ response is characteristic for each pair of odor and PN. The temporal structure of odor representation in physiological recordings from AL neurons in locust and fruit fly has been demonstrated to vary strongly with odor identity, even under constant stimulus conditions (Müller et al., 
2002; Stopfer et al., 2003; Wilson et al., 2004). Likewise, intensity has been shown to affect the temporal dynamics of $\mathrm{PN}$ responses in a characteristic way (Stopfer et al., 2003; Ito et al., 2009). A large part of the temporal complexity seems to be generated already at the level of ORNs (Raman et al., 2010). A recent modeling study has shown how downstream neurons can extract temporal relationships between the firing of populations in the locust AL (Assisi et al., 2011).

Most studies that deal with olfactory coding in the AL (including the present one) have focused on either the spatial or the temporal aspect of the spatio-temporal code while putting little emphasis on the other. This can be partly explained by the distinct experimental approaches that provide the empirical basis for model approaches. Imaging techniques monitor a spatial arrangement, often with low temporal resolution, while electrophysiological recordings of single neurons or few units provide high temporal resolution but poor spatial information. The existence of a spatial code in the honeybee has been repeatedly proven in glomerular space and is reproducible across animals (Galizia et al., 1999; Wang et al., 2003). However, glomerular response patterns have mostly been obtained by measuring $\mathrm{Ca}^{2+}$-activity, with temporal resolution typically in the range of $5 \mathrm{~Hz}$. This resolution is not sufficient to identify temporal variations in odor representation, which have been described to occur at frequencies starting at $20 \mathrm{~Hz}$ using electrophysiological methods (e.g., Stopfer et al., 1997; Laurent et al., 1998; Wilson and Laurent, 2005). On the other hand, electrophysiological studies either have very small spatial coverage, or lack the possibility to determine the exact spatial location of the signal source, e.g., in the case of local field potentials. Hence, a direct comparison of how spatial (that is, glomerular) and temporal coding interact in the insect olfactory system is still missing. Given the current experimental evidence, it is difficult to reconcile the debate whether the spatial or the temporal component of odor coding is more important in odor discrimination. It is also likely that different insect species have evolved different odor coding strategies, given the considerable difference in the anatomical layout of their

\section{REFERENCES}

Abel, R., Rybak, J., and Menzel, R. (2001). Structure and response patterns of olfactory interneurons in the honeybee, Apis mellifera. J. Comp. Neurol. 437, 363-383.

Asahina, K., Louis, M., Piccinotti, S., and Vosshall, L. B. (2009). A circuit supporting concentration-invariant odor perception in Drosophila. J. Biol. 8, 9.

Assisi, C., Stopfer, M., and Bazhenov, M. (2011). Using the structure of inhibitory networks to unravel mechanisms of spatiotemporal patterning. Neuron 69, 373-386.

Bhandawat, V., Olsen, S. R., Gouwens, N. W., Schlief, M. L., and Wilson, R. I. (2007). Sensory processing in the Drosophila antennal lobe increases reliability and separability of ensemble odor representations. Nat. Neurosci. 10, 1474-1482.
Brandstätter, A. S., and Kleineidam, C. (2011). Distributed representation of social odors indicates parallel processing in the antennal lobe of ants. J. Neurophysiol. 106, 2437-2449

Brill, M. F., Reus, I., Rosenbaum, T., Kleineidam, C. J., and Rössler, W. (2011). "Simultaneous recordings from multiple projection neurons in the dual olfactory pathway of the honeybee," Poster Abstract, in Proceedings of the 9th Göttingen Meeting of the German Neuroscience Society, Göttingen, T19-31A.

Carlsson, M. A., Chong, K. Y., Daniels, W., Hansson, B. S., and Pearce, T. C. (2007). Component information is preserved in glomerular responses to binary odor mixtures in the moth Spodoptera littoralis. Chem. Senses 32, 433-443.

olfactory systems (e.g., number of glomeruli, functional separation between structural glomeruli, single vs. dual pathway systems, multi- vs. uniglomerular PNs, see Martin et al. (2011) for a review), possibly with different emphasis on spatial and temporal encoding of odors.

In this context, it is interesting to note that in the honeybee, temporally complex responses are observed frequently in m-ACT PNs (Müller et al., 2002), but rarely in l-ACT PNs (Krofczik et al., 2009). This observation may indicate that l-ACT and m-ACT are not only different in the spatial component of odor representation, but also in the temporal component. Moreover, the m-ACT is shared between locust, Drosophila, and honeybee, while the 1ACT is unique to Hymenoptera, notably bees, and ants (Galizia and Rössler, 2010). The difference in temporal aspects of the neural code between $1-$ and $\mathrm{m}$-ACT will be a fascinating topic for future studies as simultaneous observations from PNs in both pathways with suitable temporal resolution become available (Brill et al., 2011; Rosenbaum et al., 2011).

\section{ACKNOWLEDGMENTS}

We thank the reviewers for providing valuable input. We are particularly grateful to Pawel Andrzej Herman (acting anonymously during the review process) for his numerous constructive comments which helped us to improve the manuscript considerably. This work was supported by the German ministry for education and research (Bundesministerium für Bildung und Forschung, BMBF) within the Bernstein Partner project "Olfactory coding: Integrating experimental and theoretical approaches" (grant no. 01GQ0772 to Randolf Menzel), within the Bernstein Focus "Neural Basis of Learning” (grant 01GQ0941 to Martin Paul Nawrot and Randolf Menzel), and within the Bernstein Center for Computational Neuroscience Berlin (grant no. 01GQ1001D to Michael Schmuker), by the "Innovationsfonds" from Freie Universität Berlin, and by Deutsche Forschungsgemeinschaft within the priority program SPP 1392 "Integrative analysis of olfaction" (SCHM 2474/1-1 to Michael Schmuker).

Chou, Y.-H., Spletter, M. L., Yaksi, E., Leong, J. C. S., Wilson, R. I., and Luo, L. (2010). Diversity and wiring variability of olfactory local interneurons in the Drosophila antennal lobe. Nat. Neurosci. 13, 439-449.

Deisig, N., Giurfa, M., Lachnit, H., and Sandoz, J.-C. (2006). Neural representation of olfactory mixtures in the honeybee antennal lobe. Eur. J. Neurosci. 24, 1161-1174.

Deisig, N., Giurfa, M., and Sandoz, J. C. (2010). Antennal lobe processing increases separability of odor mixture representations in the honeybee. J. Neurophysiol. 103, 2185-2194.

Flanagan, D., and Mercer, A. (1989). An atlas and 3-D reconstruction of the antennal lobes in the worker honey bee, Apis mellifera L. (Hymenoptera: Apidae). Int. J. Insect Morphol. Embryol. 18, 145-159.
Fonta, C., Sun, X., and Masson, C. (1993). Morphology and spatial distribution of bee antennal lobe interneurones responsive to odours. Chem. Senses 18, 101-119.

Galán, R. F., Sachse, S., Galizia, C. G., and Herz, A. V. M. (2004). Odor-driven attractor dynamics in the antennal lobe allow for simple and rapid olfactory pattern classification. Neural Comput. 16, 999-1012.

Galizia, C. G., and Rössler, W. (2010). Parallel olfactory systems in insects: anatomy and function. Annu. Rev. Entomol. 55, 399-420.

Galizia, C. G., Sachse, S., Rappert, A., and Menzel, R. (1999). The glomerular code for odor representation is species specific in the honeybee Apis mellifera. Nat. Neurosci. 2, 473-478. 
Ganeshina, O., and Menzel, R. (2001). GABA-immunoreactive neurons in the mushroom bodies of the honeybee: an electron microscopic study. J. Comp. Neurol. 437, 335-349.

Grünewald, B. (1999). Morphology of feedback neurons in the mushroom body of the honeybee, Apis mellifera. J. Comp. Neurol. 404, 114-126.

Hourcade, B., Muenz, T. S., Sandoz, J. C., Rössler, W., and Devaud, J. M. (2010). Long-term memory leads to synaptic reorganization in the mushroom bodies: a memory trace in the insect brain? J. Neurosci. 30, 6461-6465.

Huerta, R., Nowotny, T., GarciaSanchez, M., Abarbanel, H. D., and Rabinovich, M. (2004). Learning classification in the olfactory system of insects. Neural Comp. 16, 1601-1640.

Ito, I., Bazhenov, M., Ong, R. C., Raman, B., and Stopfer, M. (2009). Frequency transitions in odorevoked neural oscillations. Neuron 64, 692-706.

Kazama, H., and Wilson, R. I. (2008). Homeostatic matching and nonlinear amplification at identified central synapses. Neuron 58, 401-413.

Kirschner, S., Kleineidam, C. J., Zube, C., Rybak, J., Grünewald, B., and Rössler, W. (2006). Dual olfactory pathway in the honeybee, Apis mellifera. J. Comp. Neurol. 499, 933-952.

Knudsen, J., Eriksson, R., and Gershenzon, J. (2006). Diversity and distribution of floral scent. Bot. Rev. 72, 1-120.

Krofczik, S., Menzel, R., and Nawrot, M. P. (2009). Rapid odor processing in the honeybee antennal lobe network. Front. Comput. Neurosci. 2:9. doi:10.3389/neuro.10.009.2008

Laurent, G., MacLeod, K., Stopfer, M., and Wehr, M. (1998). Spatiotemporal structure of olfactory inputs to the mushroom bodies. Learn. Mem. 5, 124-132.

Linster, C., Sachse, S., and Galizia, C. G. (2005). Computational modeling suggests that response properties rather than spatial position determine connectivity between olfactory glomeruli. J. Neurophysiol. 93, 3410-3417.

Linster, C., and Smith, B. H. (1997). A computational model of the response of honey bee antennal lobe circuitry to odor mixtures: overshadowing, blocking and unblocking can arise from lateral inhibition. Behav. Brain Res. 87, 1-14.

Martin, J. P., Beyerlein, A., Dacks, A. M., Reisenman, C. E., Riffell, J. A., Lei, H., and Hildebrand, J. G. (2011). The neurobiology of insect olfaction: sensory processing in a comparative context. Prog. Neurobiol. 95, 427-447.

Meyer, A. (2011). Characterisation of Local Interneurons in the Antennal Lobe of the Honeybee. Ph.D. thesis, Department of Biology, Universität Konstanz. Konstanz.

Meyer, A., and Galizia, C. G. (2011). Elemental and configural olfactorycoding by antennal lobeneurons of the honey bee (Apis mellifera). J. Comp. Physiol. A. doi: 10.1007/s00359-011-0696-8. [Epub ahead of print].

Mobbs, P. G. (1982). The brain of the honeybee Apis mellifera. I. The connections and spatial organization of the mushroom bodies. Philos. Trans. R. Soc. Lond. B Biol. Sci. 298 309-354.

Moraud, E. M., and Martinez, D. (2010). Effectiveness and robustness of robot infotaxis for searching in dilute conditions. Front. Neurorobot. 4:1. doi:10.3389/fnbot.2010.00001

Müller, D., Abel, R., Brandt, R., Zöckler, M., and Menzel, R. (2002). Differential parallel processing of olfactory information in the honeybee, Apis mellifera L. J. Comp. Physiol. A Neuroethol. Sens. Neural Behav. Physiol. 188, 359-370.

Murlis, J. (1997). "Odor plumes and the signal they provide," in Insect Pheromone Research: New Directions, eds R. T. Cardé and A. K. Minks (New York, NY: Chapman and Hall), 221-231.

Nowotny, T., Huerta, R., Abarbanel, H. D., and Rabinovich, M. I. (2005). Self-organization in the olfactory system: one shot odor recognition in insects. Biol. Cybern. 93, 436-446.

Okada, R., Manz, G., and Menzel, R. (2007). Learning-related plasticity in PE1 and other mushroom bodyextrinsic neurons in the honeybee brain. J. Neurosci. 27, 11736-11747.

Olsen, S. R., and Wilson, R. I. (2008). Lateral presynaptic inhibition mediates gain control in an olfactory circuit. Nature 452, 956-960.

Papadopoulou, M., Cassenaer, S., Nowotny, T., and Laurent, G. (2011). Normalization for sparse encoding of odors by a wide-field interneuron. Science 332, 721-725.

Perez-Orive, J., Bazhenov, M., and Laurent, G. (2004). Intrinsic and circuit properties favor coincidence detection for decoding oscillatory input. J. Neurosci. 24, 6037-6047.

Raguso, R. A. (2008). Wake up and smell the roses: the ecology and evolution of floral scent. Annu. Rev. Ecol. Evol. Syst. 39, 549-569.

Raman, B., Joseph, J., Tang, J., and Stopfer, M. (2010). Temporally diverse firing patterns in olfactory receptor neurons underlie spatiotemporal neural codes for odors. J. Neurosci. 30, 1994-2006.

Riffell, J. A., Abrell, L., and Hildebrand, J. G. (2008). Physical processes and real-time chemical measurement of the insect olfactory environment. $J$. Chem. Ecol. 34, 837-853.

Rosenbaum, T., Brill, M., Rössler, W. and Nawrot, M. P. (2011) "Do antennal lobe output neurons employ a latency code?" Poster Abstract, in Proceedings of the 9th Göttingen Meeting of the German Neuroscience Society, Göttingen, T19-30A.

Sachse, S., and Galizia, C. G. (2002). Role of inhibition for temporal and spatial odor representation in olfactory output neurons: a calcium imaging study. J. Neurophysiol. 87, 1106-1117.

Sachse, S., and Galizia, C. G. (2003). The coding of odour-intensity in the honeybee antennal lobe: local computation optimizes odour representation. Eur. J. Neurosci. 18, 2119-2132.

Sachse, S., Rappert, A., and Galizia, C. G. (1999). The spatial representation of chemical structures in the antennal lobe of honeybees: steps towards the olfactory code. Eur. J. Neurosci. 11 3970-3982.

Schmuker, M., and Schneider, G. (2007). Processing and classification of chemical data inspired by insect olfaction. Proc. Natl. Acad. Sci. U.S.A. 104, 20285-20289.

Schmuker, M., Schwarte, F., Brück, A., Proschak, E., Tanrikulu, Y., Givehchi, A., Scheiffele, K., and Schneider, G. (2006). SOMMER: self-organising maps for education and research. $J$. Mol. Model. 13, 225-228.

Seki, Y., Rybak, J., Wicher, D., Sachse, S., and Hansson, B. S. (2010). Physiological and morphological characterization of local interneurons in the Drosophila antennal lobe. J. Neurophysiol. 104, 1007-1019.

Sigma-Aldrich. (2004). Flavors and Fragrances. Milwaukee, WI: SigmaAldrich.

Silbering, A. F., and Galizia, C. G. (2007). Processing of odor mixtures in the Drosophila antennal lobe reveals both global inhibition and glomerulus-specific interactions. J. Neurosci. 27, 11966-11977.

Silbering, A. F., Okada, R., Ito, K., and Galizia, C. G. (2008). Olfactory information processing in the Drosophila antennal lobe: anything goes? J. Neurosci. 28, 13075-13087.

Stopfer, M., Bhagavan, S., Smith, B. H., and Laurent, G. (1997). Impaired odour discrimination on desynchronization of odour-encoding neural assemblies. Nature 390, 70-74.

Stopfer, M., Jayaraman, V., and Laurent, G. (2003). Intensity versus identity coding in an olfactory system. Neuron 39, 991-1004.

Strube-Bloss, M. F., Nawrot, M. P., and Menzel, R. (2011). Mushroom body output neurons encode odorreward associations. J. Neurosci. 31, 3129-3140.

Tabor, R., Yaksi, E., Weislogel, J.-M., and Friedrich, R. W. (2004). Processing of odor mixtures in the zebrafish olfactory bulb. J. Neurosci. 24, 6611-6620.

Thistle, H. W., Peterson, H., Allwine, G., Lamb, B., Strand, T., Holsten, E. H., and Shea, P. J. (2004). Surrogate pheromone plumes in three forest trunk spaces: composite statistics and case studies. For. Sci. 50, 610-625.

Vergassola, M., Villermaux, E., and Shraiman, B. I. (2007). "Infotaxis" as a strategy for searching without gradients. Nature 445, 406-409.

Vickers, N. J. (2000). Mechanisms of animal navigation in odor plumes. Biol. Bull. 198, 203-212.

Wang, J. W., Wong, A. M., Flores, J., Vosshall, L. B., and Axel, R. (2003). Two-photon calcium imaging reveals an odor-evoked map of activity in the fly brain. Cell 112, 271-282.

Wick, S. D., Wiechert, M. T., Friedrich, R. W., and Riecke, H. (2010). Pattern orthogonalization via channel decorrelation by adaptive networks. J. Comp. Neurosci. 28, 29-45.

Wilson, R. I., and Laurent, G. (2005). Role of GABAergic inhibition in shaping odor-evoked spatiotemporal patterns in the Drosophila antennal lobe. J. Neurosci. 25, 9069-9079.

Wilson, R. I., Turner, G. C., and Laurent, G. (2004). Transformation of olfactory representations in the Drosophila antennal lobe. Science 303, 366-370.

Wright, G. A., Lutmerding, A., Dudareva, N., and Smith, B. H. (2005). Intensity and the ratios of compounds in the scent of snapdragon flowers affect scent discrimination by honeybees (Apis mellifera). J. Comp. Physiol. A Neuroethol. Sens. Neural Behav. Physiol. 191, 105-114.

Wright, G. A., Skinner, B. D., and Smith, B. H. (2002). Ability of honeybee, Apis mellifera, to detect and discriminate odors of varieties of canola (Brassica rapa and Brassica napus) and snapdragon flowers (Antirrhinum majus). J. Chem. Ecol. 28, 721-740. 
Yamagata, N., Schmuker, M., Szyszka, P., Mizunami, M., and Menzel, R. (2009). Differential odor processing in two olfactory pathways in the honeybee. Front. Syst. Neurosci. 3:16. doi:10.3389/neuro.06.016.2009

Zollner, G. E., Torr, S. J., Ammann, C., and Meixner, F. X. (2004). Dispersion of carbon dioxide plumes in African woodland: implications for host-finding by tsetse flies. Physiol. Entomol. 29, 381-394.

Conflict of Interest Statement: The authors declare that the research was conducted in the absence of any commercial or financial relationships that could be construed as a potential conflict of interest.
Received: 05 September 2011; paper pending published: 20 September 2011; accepted: 01 December 2011; published online: 28 December 2011.

Citation: Schmuker M, Yamagata $N$ Nawrot MP and Menzel R (2011) Parallel representation of stimulus identity and intensity in a dual pathway model inspired by the olfactory system of the honeybee. Front. Neuroeng. 4:17. doi: 10.3389/fneng.2011.00017

Copyright (C) 2011 Schmuker, Yamagata, Nawrot and Menzel. This is an openaccess article distributed under the terms of the Creative Commons Attribution Non Commercial License, which permits non-commercial use, distribution, and reproduction in other forums, provided the original authors and source are credited. 